In a study by Tabbara and Al-Hemidan, 10 patients with retinal vasculitis associated with Behçet disease who did not respond to conventional immunosuppressive treatment received a total of six infusions of infliximab $5 \mathrm{mg} / \mathrm{kg}$. A retrospective comparison with 33 patients who received conventional therapy found that those treated with infliximab experienced fewer relapses and ocular complications, improved visual acuity and less inflammation over the 2-3-year follow-up period. Lopez-Gonzalez et al. reported the 7-year results of infliximab treatment in a review of 11 patients with Behçet disease and other inflammatory eye diseases refractory to conventional therapy. In this longterm study, visual acuity was maintained or improved in most patients, and inflammation was reduced in all patients following treatment with infliximab, although no effect on chorioretinal lesions was noted.

Rapid neutralization of tumor necrosis factor with infliximab could prevent vision-threatening damage caused by inflammation, although future studies will need to determine whether this treatment can be used as prophylactic therapy.

Original articles Tabbara KF and Al-Hemidan AI (2008) Infliximab effects compared to conventional therapy in the management of retinal vasculitis in Behçet disease. Am J Ophthalmol 146: 845-850

Lopez-Gonzalez R et al. (2008) Treatment of refractory posterior uveitis with infliximab: a 7-year follow-up study. Scand J Rheumatol 5: 1-5

\section{Use of PET with macrophage imaging to detect joint inflammation in RA}

PET to detect joint inflammation in rheumatoid arthritis (RA) is usually performed with ${ }^{18} \mathrm{~F}$-fluorodeoxyglucose, a tracer of glucose metabolism. As increased glucose metabolism is not a specific indicator of inflammation, however, van der Laken et al. investigated the use of PET with ${ }^{11} \mathrm{C}-(R)-\mathrm{PK} 11195$, a macrophage targeting tracer that binds to peripheral benzodiazepine receptors on macrophages, in patients with RA.

The authors found a high correlation between tracer uptake and clinically determined synovial inflammation, with significantly greater uptake seen in knees with severe swelling compared with those with mild or no swelling. Immunohistochemical staining of 11 samples of inflamed synovial tissue from 6 patients seemed to confirm that uptake was due to binding of the PK11195 ligand to macrophage receptors. In addition, scans from the contralateral, noninflamed knees of patients with RA showed significantly increased tracer uptake compared with control knees of patients without inflammatory joint disease. As macrophage infiltration might be an early indicator of RA, the authors suggest that ${ }^{11} \mathrm{C}-(R)-\mathrm{PK} 11195$ PET has potential for use in the detection of subclinical joint inflammation. Advantages of the technique include the short half-life of carbon-11 and the possibility of reducing the duration of the procedure from $1 \mathrm{~h}$ to $20 \mathrm{~min}$.

This is the first, albeit small, study of PET using a macrophage tracer to detect joint inflammation in patients with RA; further investigations into the potential of this technique are warranted.

Original article van der Laken CJ et al. (2008) Noninvasive

imaging of macrophages in rheumatoid synovitis using

${ }^{11} \mathrm{C}-(R)-\mathrm{PK} 11195$ and positron emission tomography.

Arthritis Rheum 58: 3350-3355

\section{Distinct clinical features of polymyalgia rheumatica}

Polymyalgia rheumatica (PMR) and seronegative late-onset rheumatoid arthritis (RA) share a number of immunogenetic and phenotypic features at disease onset, which can be problematic for the diagnosis and management of these diseases. A 5-year prospective study has identified clinical features that could distinguish the two disorders at presentation.

Pease et al. recruited 88 patients with rheumatoid factor (RF)-negative late-onset RA and 189 patients with PMR. After correction for multiple variables, clinical evidence of arthritis in the wrist and proximal interphalangeal joints (with or without metacarpophalangeal joint involvement) at presentation was significantly more common in patients with seronegative lateonset RA than in those with PMR. In addition, RF-negative late-onset RA was associated with a lower plasma viscosity (a direct correlate of erythrocyte sedimentation rate) at onset than PMR; however, the viscosity normalized with treatment in PMR, whereas it remained high in late-onset RA. The distribution of HLA-DRB1 alleles was similar in both groups. Patients with PMR who developed arthritis responded more rapidly to corticosteroids, with a lower rate of arthritis recurrence, than those with 